\title{
Do gender, disability, and morbidity affect aging rate in the LLFS? Application of indices of cumulative deficits
}

\author{
Alexander M. Kulminski ${ }^{\text {a,* }}$, Konstantin G. Arbeev ${ }^{\text {a }}$, Kaare Christensen ${ }^{\mathrm{b}}$, Richard Mayeux ${ }^{\mathrm{c}}$, \\ Anne B. Newman ${ }^{d}$, Michael A. Province ${ }^{e}$, Evan C. Hadley ${ }^{f}$, Winifred Rossi ${ }^{f}$, Thomas T. Perls ${ }^{g}$, \\ Irma T. Elo ${ }^{\mathrm{h}}$, Anatoli I. Yashin ${ }^{\mathrm{a}}$ \\ ${ }^{a}$ Duke University, Center for Population Health and Aging, Durham, NC 27708-0408, USA \\ ${ }^{\mathrm{b}}$ The Danish Aging Research Center, University of Southern Denmark, 5000 Odense C, Denmark \\ ${ }^{c}$ Columbia University, Health Sciences Division, 630 West 168th Street PS 1-146, New York 10032, USA \\ ${ }^{\mathrm{d}}$ University of Pittsburgh, Division of Geriatric Medicine, 3459 Fifth Avenue, 4th Floor, Pittsburgh, PA 15213, USA \\ e Washington University School of Medicine, Division of Statistical Genomics Box 8506, 4444 Forest Park Boulevard, St. Louis, MO 63108, USA \\ ${ }^{\mathrm{f}}$ National Institute on Aging, Building 31, Room 5C27, 31 Center Drive, MSC 2292, Bethesda, MD 20892, USA \\ ${ }^{\mathrm{g}}$ Boston University, School of Medicine, Division of Geriatrics - MED Geriatrics, 715 Albany St, B, Boston, MA 02215, USA \\ h Irma T. Elo, 232 McNeil Building 3718 Locust Walk University of Pennsylvania, Sociology Department, Philadelphia, PA 19104-6298, USA
}

\section{A R T I C L E I N F O}

\section{Article history:}

Received 26 January 2011

Received in revised form 7 March 2011

Accepted 23 March 2011

Available online 2 April 2011

\section{Keywords:}

Aging rates

Longevity

Cumulative indices

Long-Life Family Study

\begin{abstract}
A B S T R A C T
We used an approach of cumulative deficits to evaluate the rate of aging in 4954 participants of the Long-Life Family Study (LLFS) recruited in the U.S. (Boston, New York, and Pittsburg) and Denmark. We used an array of 85 health-related deficits covering major health dimensions including depression, cognition, morbidity, physical performance, and disability to construct several deficit indices (DIs) with overlapping and complementary sets of deficits to test robustness of the estimates. Our study shows that the DIs robustly characterize accelerated rates of aging irrespective of specific of deficits. When a wider spectrum of health dimensions is considered these rates are better approximated by quadratic law. Exponential rates are more characteristic for more severe health dimensions. The aging rates are the same for males and females. Individuals who contracted major diseases and those who were free of them exhibited the same aging rates as characterized by the DI constructed using mild deficits. Unlike health, disability can qualitatively alter the aging patterns of the LLFS participants. We report on systemic differences in health among the LLFS centenarians residing in New York and Boston. This study highlights importance of aggregated approaches to better understand systemic mechanisms of health deterioration in long-living individuals.
\end{abstract}

(c) 2011 Elsevier Ireland Ltd. All rights reserved.

\section{Introduction}

Examination of various studies on different aspects of aging, health, and longevity reveals that only a small part of the information collected in longitudinal or cross-sectional datasets is used for the respective analyses. Typically, these are variables showing strong effects on the characteristics of interest (e.g., survival, healthy life span) such as morbidity (i.e., various diseases), disease risk factors (e.g., physiological indicators), or/ and disability (i.e., impairments in activities of daily living (Katz and Akpom, 1976; Lawton and Brody, 1969)). Meanwhile, many

\footnotetext{
Abbreviations: LLFS, Long-Life Family Study; DI, deficit index.

* Corresponding author at: Center for Population Health and Aging, Duke University, Box 90408, Trent Hall, Room 002, Durham, NC 27708, USA. Tel.: +1 919684 4962; fax: +1 9196843861.

E-mail address: Alexander.Kulminski@duke.edu (A.M. Kulminski).
}

other variables each of which may not necessarily have a strong effect on adverse aging-associated outcomes are typically ignored. This strategy is not entirely convincing because many characteristics collected in such studies carry information on aging-related processes. Collectively, these variables can better characterize chances to live long (and healthy) lives than individual traits. To take advantage of rich information on various health-related traits, called here as health deficits, the indices of cumulative deficits (DIs) or frailty indices have been developed (Mitnitski et al., 2001; Rockwood et al., 2005). It has been found, that these indices provide researchers with a convenient tool for describing static and dynamic properties of the process of aging-related deterioration in human health and well-being, including initial stages of this process and its last phase (e.g., geriatric frailty state, mortality). Specifically, numerous studies in various samples documented the ability of the DI to characterize the overall and health-domain specific health deterioration, survival, hospitalization, chances to live a long life as well as the aging-related health decline (Goggins 
et al., 2005; Gu et al., 2009; Kulminski et al., 2007a,b; Mitnitski et al., 2007; Rockwood et al., 2006; Woo et al., 2006; Yashin et al., 2007a,b).

Given promising statistical properties of the DI and similar cumulative indices (Fried et al., 2009; Yashin et al., 2010), the DI can be used as a proxy of longevity and be an alternative characteristic of the aging rate (which is, for instance, measured by demographers using information on survival or mortality (Vaupel, 2010)). Indeed, one advantage of the DI is that it can robustly characterize the rate of health deterioration in aging individuals and, thus, be predictive of longevity (Kulminski et al., 2008b). Once this property is rigorously tested in numerous populations, the DI can be used as intermediate phenotype of longevity to characterize the aging rate in populations with an unknown life span. It was found that to characterize the overall health and well-being status and the aging-related decline, specifics of deficits appears to be considerably less important than the number of health-related problems a person has accumulated by a given age (Goggins et al., 2005; Kulminski et al., 2006, 2007a,b; Mitnitski et al., 2005; Rockwood et al., 2006; Yashin et al., 2007a,b). This property allows straightforward generalization of the DI strategy to different studies with a sufficiently wide set of measured individual aging-related traits.

Until recently, the approach of cumulative deficits was used either in samples of general populations or in populations with certain health problems (e.g., with disability, mental illness). In this study we focus on a unique population of about 5000 American and Danish participants of the Long-Life Family Study (LLFS) who were selected to have a high chance to live long lives, i.e., who are presumably in better health compared to general populations (Newman et al., 2011). Given this focus, most study participants are alive. The major goal is to evaluate the rate of aging defined as a rate of accumulation of health deficits and characterized by a set of DIs in this long-living population and elucidate whether these rates are altered by gender, disability, or morbidity.

\section{Data and methods}

The Long-Life Family Study (LLFS) consists of families selected for exceptional familial longevity in the United States (Boston, New York, and Pittsburg) and Denmark. The study eligibility criteria were described elsewhere (Pedersen et al., 2006; Sebastiani et al., 2009; Yashin et al., 2010). Briefly, in the United States, the family eligible for the LLFS must have two living siblings aged $80+$ years, two living offspring of one or more of the siblings, and a living spouse of one of the offspring who were considered as controls. In addition, the family must demonstrate exceptional longevity based on a Family Longevity Selection Score, which is a summary-measure based on the survival experience of the oldest living generation of siblings relative to what would be expected based on birth cohort life tables (Sebastiani et al., 2009). Families with members of this generation who are still alive and larger sibships are given higher priorities. Finally, an eligible family is enrolled in the LLFS if at least 3 family members (the proband, at least one sibling of the proband, and one offspring of the proband or the sibling) indicate their willingness to participate.

In Denmark, the identification of potentially eligible probands and their families is as follows. First, individuals who would be aged $90+$ years during the study recruitment period are identified in the Danish National Register of Persons. Second, using information on the place of birth and the names, parish registers available in regional archives are searched to locate the parents of the elderly individuals in order to identify sibships. Based on the above information, potentially eligible families are identified and contact is made with potential probands to further assess the family's eligibility for and willingness to participate in the LLFS using criteria parallel to that used in the United States.

Once enrolled in the LLFS, information from the US and Danish participants was collected using similar questionnaires and in-home physical examinations, covering such topics as socio-demographic characteristics, physical activity and functioning, health and medical history, cognitive functioning, mood and personality, anthropometry, blood pressure, and spirometry. In this study, we use data on 1271 Danish subjects and 3683 US subjects who had completed most the data collection on April 9, 2010.

Indices of cumulative deficits are conceptualized as the proportion of health traits that occurred by age $x$ (Kulminski et al., 2008b; Mitnitski et al., 2001). For this study we selected all 89 ordinal deficits available in the study covering major health dimensions including depression, cognition, morbidity, physical performance, disability, etc. Four deficits were redundant due to high correlation (Pearson two-tailed correlation coefficient was 0.5 or larger) and, thus, these were dropped. Dichotomous deficits were re-coded as 1 (there is a deficit) and 0 (no deficit). Non-dichotomous deficits were rescaled to the unit interval according to severity of the trait (e.g., $0=$ no problem, $0.5=$ sometime, $1=$ yes problem). Disability state was approximated as having one or more of the six disability-related deficits (Table 1). Morbidity status

Table 1

The 85 deficits selected for construction of the DIs and characterization of disability and morbidity

\begin{tabular}{|c|c|c|}
\hline \multicolumn{2}{|c|}{58 deficits excluding those characterizing disability and morbidity } & \multirow{2}{*}{$\begin{array}{l}\text { Disabilities } \\
\text { Confinement to bed }\end{array}$} \\
\hline Bothered & Hip fractures & \\
\hline Trouble keeping mind & Wrist fractures & In/out of bed/chairs \\
\hline Everything was an effort & Spine fractures & Bathing/showering \\
\hline Felt depressed & Other fractures & Walking in small room \\
\hline Felt hopeless & Fallen & Walking $1 / 4$ mile \\
\hline Felt fearful or anxious & Hospitalized & Walking 1 flight stairs \\
\hline Sleep was restless & Other health problems & \\
\hline Was unhappy & Any walking in past 2 weeks & Morbidity \\
\hline Felt lonely & Regularly walk $1+$ hours & Myocardial Infarction \\
\hline Could not get going & Lack of vigorous exercise & Heart Failure \\
\hline A worrier & Current eyesight & Stroke \\
\hline MMSE Score & Current hearing ability & Asthma \\
\hline Ability to see & Difficulty to walk $1 / 4$ mile & Chronic bronchitis \\
\hline Ability to hear & Pain in right hand & Emphysema/COPD \\
\hline Ability to speak & Side-by-side stand & Pneumonia \\
\hline Coronary angioplasty & Semi-tandem stand & Pulmonary fibrosis \\
\hline Atrial fibrillation & Tandem stand & Diabetes \\
\hline Deep vein thrombosis & Not safe to stand from a chair & Thyroid disease \\
\hline Rheumatic fever & Not safe to stand from a chair 5 times & Osteoporosis \\
\hline High blood pressure & Cough most days & Liver Disease \\
\hline Transient ischemic attack or mini-stroke ${ }^{\mathrm{a}}$ & Phlegm from chest & Kidney disease \\
\hline Chest surgery & Use oxygen therapy & Alzheimer disease \\
\hline Arthritis & Chest wheezy when cold & Parkinson disease \\
\hline Depression & Chest sound wheezy most days & Blood cancer \\
\hline Melanoma & Chest wheezy most & Colon/rectal cancer \\
\hline Other skin cancers & Recent surgery & Lung cancer \\
\hline Cataract surgery 1 eye & Problem doing spirometry & Esophageal cancer \\
\hline Macular degeneration & Respiratory infection & Pancreatic cancer \\
\hline Glaucoma & Inhalers & Other cancers \\
\hline
\end{tabular}

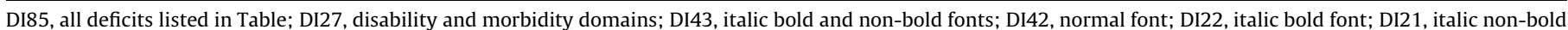
font.

a TIA, transient ischemic attack or mini-stroke. 


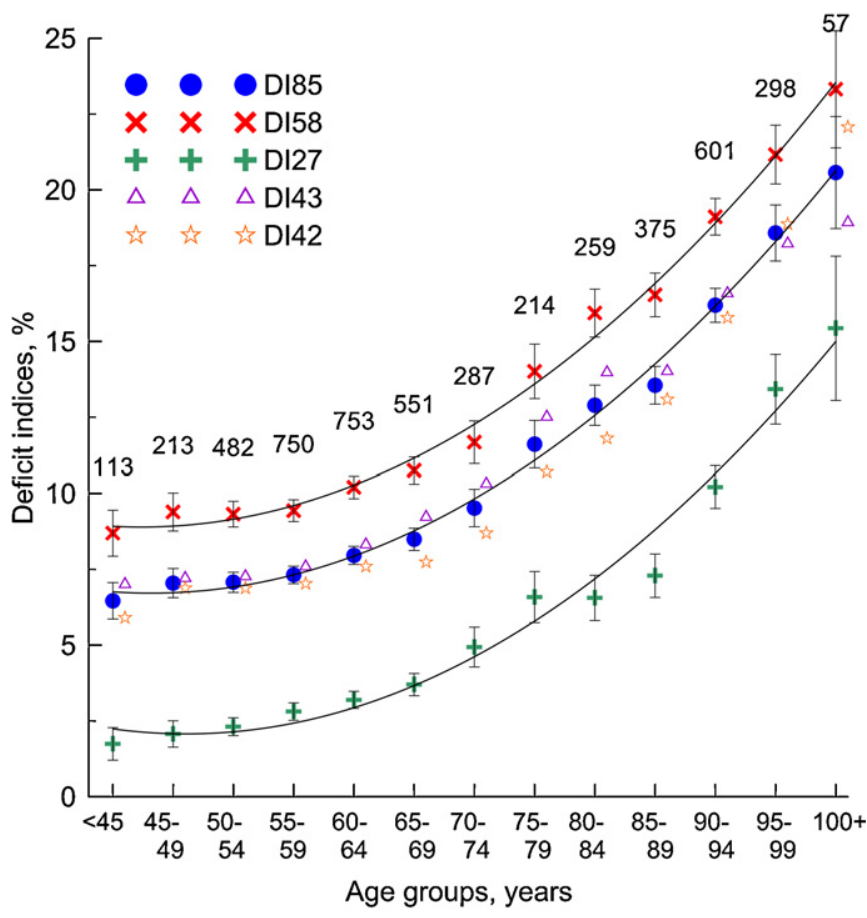

Fig. 1. Age patterns of the DIs constructed using overlapping and complementary sets of deficits. The number of deficits used for construction of the DIs is indexed next to the DI in the inset. Numbers over bars show the number of individuals in each age group. Bars show 95\% confidence interval for mean DI for each age group. Solid lines depict quadratic fits with parameters shown in Table 2.

(i.e., "healthy" vs. "unhealthy") was determined as having at least one of 21 major diseases (Table 1). DIs are constructed as percentage of heath traits and, thus, they theoretically range between $0 \%$ (good health) and $100 \%$ (poor health). We constructed three DIs using: (i) all 85 deficits (DI85), (ii) 58 of 85 deficits which were not used to characterize disability and morbidity (DI58), and (iii) 27 of 85 deficits characterizing disability and morbidity states (DI27); these deficits were not included in DI58. We also constructed two pairs of DIs using smaller number of deficits which did not overlap in each pair. Deficits for each DI pair were randomly selected from each health domain to cover the entire health spectrum. One pair of DIs was constructed using complementary fractions of deficits from the DI85, i.e., DI43 (43 of 85 deficits, Table 1 italic font) and DI42 (remaining 42 of 85 deficits not included in DI43). The other pair was constructed using complementary fractions of deficits from the DI43, i.e., DI22 (22 of 43 deficits, Table 1, italic bold font) and DI21 ( 21 of 43 deficits not included in DI22). These DIs were used to test robustness of the DI concept in the LLFS study.

\section{Results}

Fig. 1 shows remarkably consistent accelerated age patterns of DIs constructed using overlapping and complementary sets of different deficits. Table 2 shows that these patterns are mostly quadratic. They are more robust and are better approximated by quadratic function when a larger number of deficits from different health dimensions is considered (Table $2, R^{2}$ ). In most cases quadratic rates $\left(a_{2}\right)$ do not differ significantly across DIs showing highly significant increase in the rate of accumulation of deficits per year, e.g., $a_{2}=4.31 \times 10^{-3} \%$ for DI85. When a smaller number of deficits is considered, however, specifics of deficits can be more pronounced resulting in significant differences in the acceleration rate (see non-overlapping 95\% Confidence intervals [CIs] for $a_{2}$ for DI43 and DI42 in Table 2). Furthermore, accumulation of deficits from particular health dimensions (e.g., morbidity and disability in the case of DI27) might be better considered with exponential fits, although the difference from a quadratic fit in the case of DI27 (Table $2, R^{2}$ ) is not important. No gender differences in the age patterns were observed (Table 2, DI58 and DI27).

A surprising observation is that both healthy and unhealthy individuals accumulate deficits not associated with morbidity and disability (i.e., characterized by DI58) in identical quadratic fashion (Fig. 2A). Identical exponential increase in the rates is also observed for disabled and non-disabled individuals (Fig. 2B), although in this case the fits are not as good as in Fig. 2A. Careful inspection of Fig. 2B reveals that non-disabled individuals actually accumulate deficits at a strongly accelerated exponential rate $\left(R^{2}=0.985 ; a_{1}=0.083 ; 95 \% \mathrm{CI}=0.054-0.112\right)$ until certain old age (about 80 years in this case). Accumulation of deficits decelerates al the oldest-old ages (80+). This different dynamics explains the poor exponential fit of the entire age pattern.

Analysis of the age patterns of DI27 (Fig. 3), which characterizes disability/morbidity-related health dimensions, reveals qualitatively similar peculiarities for the samples of healthy/unhealthy and disabled/non-disabled individuals. Specifically, healthy individuals (i.e., who, by definition, are free of major diseases) and unhealthy individuals accumulate DI27 deficits in very similar fashion which is, however, better attributed to the exponential law (Fig. 3A). Unlike Fig. 2A, however, healthy individuals in this case tend to accumulate (disability-related) deficits significantly more rapidly than the unhealthy ones (Fig. 3A, note non-overlapping CIs for $\left.a_{1}\right)$.

Non-disabled individuals (Fig. 3B) accumulate major diseases (as defined by the DI27) following exponential pattern up to about 75 years $\left(R^{2}=0.998 ; a_{1}=0.030 ; 95 \% \mathrm{CI}=0.028-0.031\right)$. At older ages this pattern levels off and even declines. Disabled individuals accumulate deficits (i.e., diseases and disabilities) with the same rate as in the case of Fig. 2B, i.e., for exponential fit $\left(R^{2}=0.795\right)$ the rate is $a_{1}=0.013$ (95\% $\left.\mathrm{CI}=0.008-0.018\right)$ which is virtually the same as in Fig. 2A. This pattern is a convolution of disease- and disability-specific deficits which show different dynamics at

Table 2

Fits to age patterns of all constructed deficit indices.

\begin{tabular}{|c|c|c|c|c|c|}
\hline Deficit indices & Sample & $R^{2}$ & Const $(95 \% \mathrm{CI})$ & $a_{1}(95 \% \mathrm{CI}) \times 10^{2}$ & $a_{2}(95 \% \mathrm{CI}) \times 10^{3}$ \\
\hline DI85 & All $^{\mathrm{b}}$ & 0.995 & $6.76(6.17,7.35)$ & $-2.71(-7.27,1.84)$ & $4.31(3.58,5.04)$ \\
\hline \multirow[t]{3}{*}{ DI58 } & All & 0.994 & $8.92(8.23,9.62)$ & $-2.06(-7.44,3.31)$ & $4.41(3.54,5.27)$ \\
\hline & Male & 0.963 & $8.84(7.22,10.46)$ & $-1.66(-14.21,10.89)$ & $4.17(2.15,6.19)$ \\
\hline & Female & 0.987 & $9.01(7.98,10.04)$ & $-2.51(-10.54,5.53)$ & $4.65(3.35,5.93)$ \\
\hline DI27 & All & 0.980 & $2.24(1.13,3.35)$ & $-5.40(-13.99,3.19)$ & $4.45(3.06,5.83)$ \\
\hline \multirow[t]{3}{*}{$\mathrm{DI} 27^{\mathrm{a}}$} & All & 0.988 & $1.64(1.49,1.80)$ & $3.65(3.39,3.92)$ & - \\
\hline & Male & 0.958 & $1.47(1.24,1.74)$ & $3.48(3.00,3.96)$ & - \\
\hline & Female & 0.994 & $1.79(1.67,1.92)$ & $3.68(3.49,3.88)$ & - \\
\hline DI43 & All & 0.985 & $6.65(5.72,7.58)$ & $4.95(-2.23,12.13)$ & $2.80(1.64,3.95)$ \\
\hline DI42 & All & 0.990 & $6.84(5.94,7.75)$ & $-10.11(-17.11,-3.12)$ & $5.75(4.63,6.88)$ \\
\hline DI22 & All & 0.986 & $7.72(6.85,8.58)$ & $1.63(-5.10,8.35)$ & $3.16(2.08,4.24)$ \\
\hline DI21 & All & 0.952 & $5.51(3.73,7.30)$ & $8.67(-5.14,22.48)$ & $2.37(0.15,4.59)$ \\
\hline
\end{tabular}

Quadratic function is of the form $\mathrm{DI}=$ const $+a_{1}($ age -40$)+a_{2}(\text { age }-40)^{2}$ with age origin rescaled to 40 years

${ }^{a}$ This pattern was fitted by exponential function of the form $\mathrm{DI}=$ const $\exp \left\{a_{1}(\right.$ age -40$\left.)\right\}$.

b All, males and females; CI, confidence interval; $R^{2}$, coefficient of determination. 

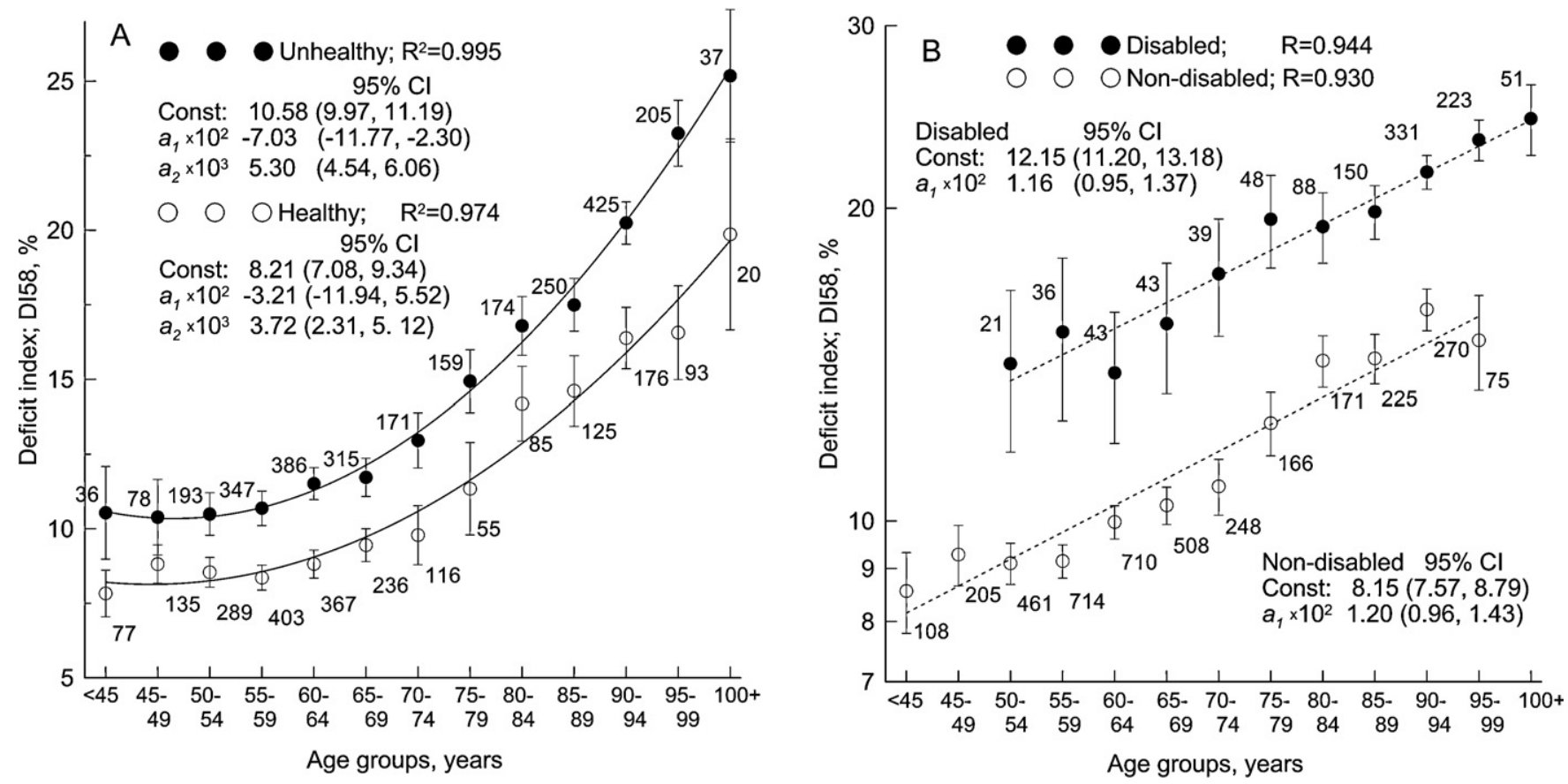

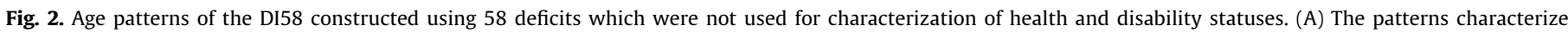

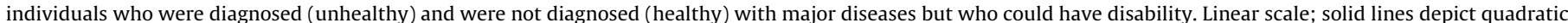

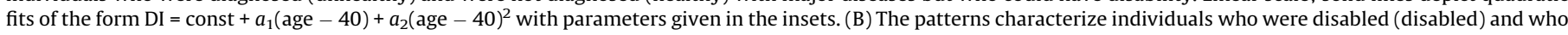

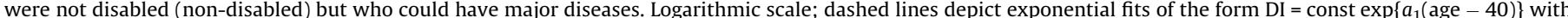

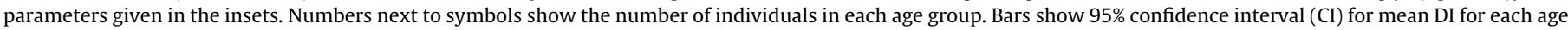
group. $R^{2}$ is coefficient of determination.

different ages. Indeed, at younger ages (less than about 75 years) the exponential increase is largely driven by morbidity (Fig. 3B, non-disabled) whereas at older ages (75+) this increase is largely attributed to the exponential increase of disability-related deficits (Fig. 3A, healthy). Deceleration of accumulation of diseases at older ages might also explain surprisingly slower aging rate for unhealthy individuals than for healthy ones (Fig. 3A).

Given the potential of the constructed DIs to characterize agingrelated health deterioration in human organism, we have examined if the DI age patterns for different members of the
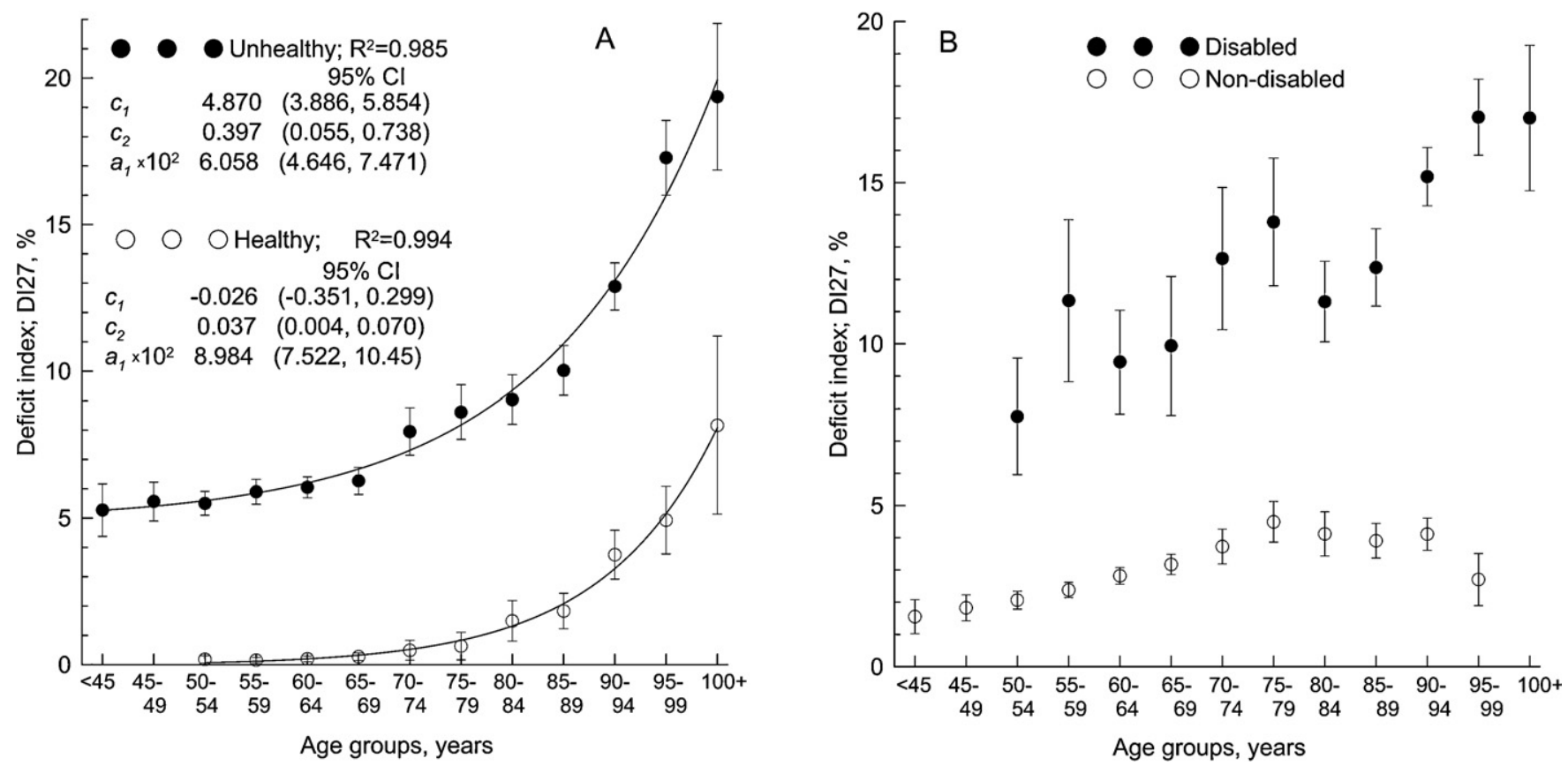

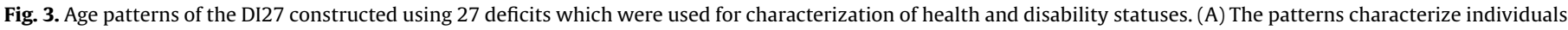

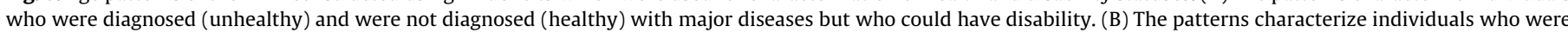

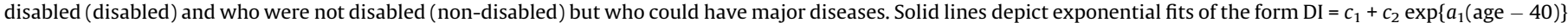

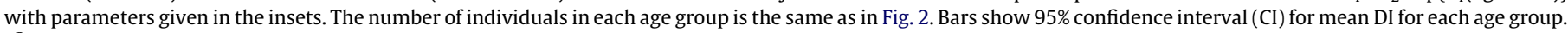
$R^{2}$ is coefficient of determination. 

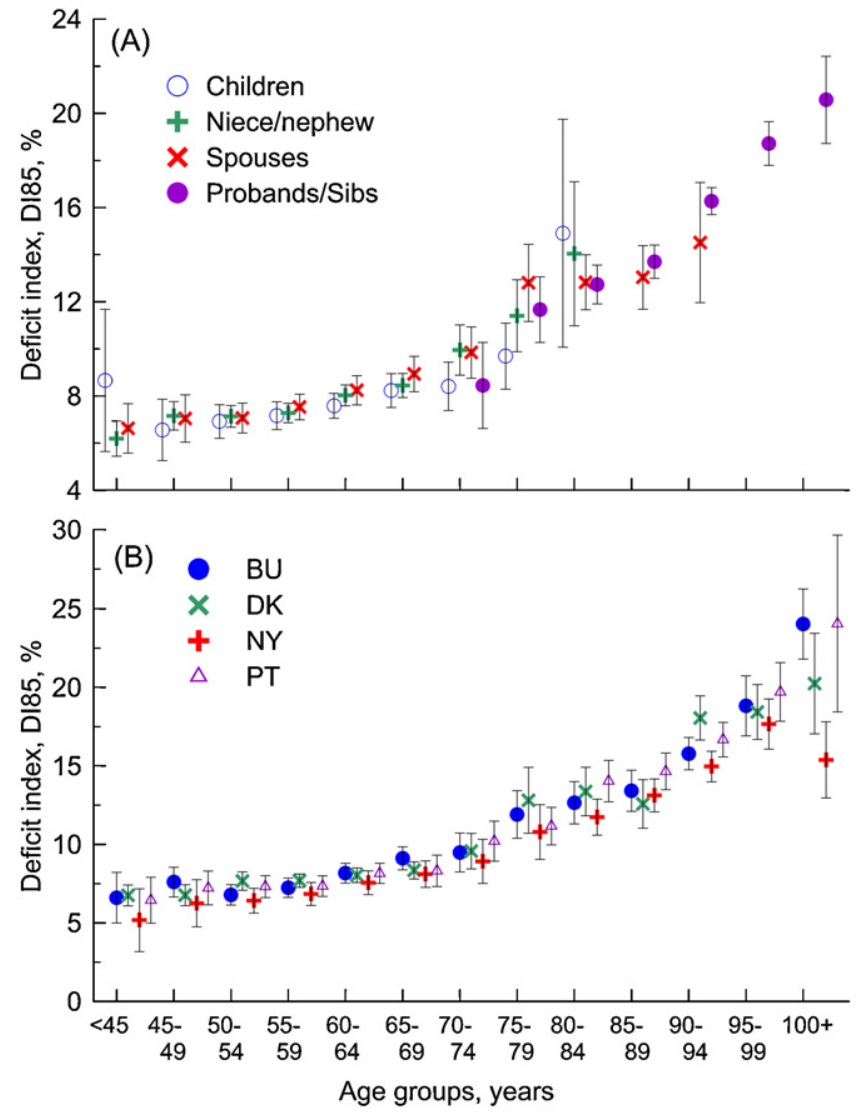

Fig. 4. Age patterns of the DI85 constructed using the entire set of 85 deficits. (A) Patterns for probands' family members. (B) Patterns for the LLFS participants residing in Denmark (DK), Boston (BU), New York (NY), and Pittsburg (PT). Bars show 95\% confidence intervals (CIs) for mean DI85 for each age group.

proband families are different. The sample includes 546 probands, 952 siblings, 71 spouses of probands and siblings, 776 children of probands, 1666 nieces and nephews, and 935 spouses of offspring (for eight individuals information was missing). First we examined age patterns for each family group. Given lack of difference in age patterns for spouses, they were aggregated into one group. Due to the same reason, we aggregated probands and siblings. Fig. 4A shows representative patterns characterized by the most comprehensive set of 85 deficits, i.e., by the DI85. Although children of probands tend to have lower aging rate compared to spouses (up to age 75 years), no apparent differences in the age patterns are seen. More detail comparison for older groups is limited by the sample size. When age-aggregated samples are considered, children of probands have better health profile defined by DI85 compared to nieces/nephews and spouses of children and nieces/nephews. Specifically, we evaluated linear trend of mean value of DI85, adjusted for sex and age, across three categories of the dependent variable characterizing relatedness, i.e., children vs. nieces/ nephews vs. their spouses. This trend was modest but highly significant (beta $=0.45, p=2.2 \times 10^{-5}$ ) implying that in the ageaggregated sample mean DI85 was the smallest (better health) among children of probands and the largest among spouses of children/nieces/nephews.

Finally, we evaluated whether members of the proband families enrolled in the LLFS at different sites experience the same aging rates or not. Given no apparent differences in age patterns for different family members, these analyses were performed for aggregated samples. Fig. 4B shows patterns of the same index, DI85, for subjects residing in Denmark (DK) and in the area covered by the three field centers in the U.S., i.e., Boston (BU), New York
Table 3

Systemic differences in health of the LLFS centenarians residing in Boston and New York are characterized by each deficit index constructed using distinct sets given in Table 1.

\begin{tabular}{llll}
\hline Deficit indices & Boston, $n=17$ & New York, $n=18$ & P-value \\
\cline { 2 - 3 } & Mean \pm SE, \% & Mean \pm SE, \% & \\
\hline DI85 & $24.0 \pm 1.1$ & $15.4 \pm 1.2$ & $1.29 \times 10^{-5}$ \\
DI58 & $26.7 \pm 1.4$ & $18.2 \pm 1.4$ & $1.14 \times 10^{-4}$ \\
DI27 & $19.5 \pm 1.7$ & $9.9 \pm 1.7$ & $3.23 \times 10^{-4}$ \\
DI43 & $21.4 \pm 1.5$ & $14.4 \pm 1.4$ & $1.46 \times 10^{-3}$ \\
DI42 & $26.4 \pm 1.0$ & $16.4 \pm 1.4$ & $2.35 \times 10^{-6}$ \\
DI22 & $23.6 \pm 2.3$ & $16.3 \pm 1.6$ & $1.23 \times 10^{-2}$ \\
DI21 & $19.0 \pm 1.6$ & $12.2 \pm 1.7$ & $6.49 \times 10^{-3}$ \\
\hline
\end{tabular}

(NY), and Pittsburg (PT). Although all subjects tend to accumulate deficits with the same rate, the level of health deterioration is significantly different in two specific age categories, i.e., between DK and NY participants aged 90-94 years and between BU and NY centenarians. Difference between DK and NY subjects is attributed to significantly higher levels of disability/morbidity characterized by DI27 for DK subjects, i.e., DI $27=13.3 \%$ (standard error $[S E]=0.95)$ for DK subjects compared to DI27 = 9.3\% $(S E=0.61)$ for NY subjects. Surprisingly, centenarians residing in New York show significantly smaller levels of health deterioration compared to centenarians residing in Boston measured by any deficit index (Table 3). Because the DI pairs (i.e., DI58 and DI27, DI43 and DI42, DI22 and DI21) were constructed using non-overlapping sets of deficits, this difference appears to be of systemic nature but not deficit-specific.

\section{Discussion and conclusions}

This study uses an approach of cumulative deficits (Kulminski et al., 2006; Mitnitski et al., 2001) to evaluate the rate of aging characterized by DIs constructed using overlapping and nonoverlapping sets of deficits associated with wide spectrum of health dimensions in successful population of long-living families. We show that these DIs robustly characterize accelerated rates of aging of about the same magnitude irrespective of specific of deficits. Similar accelerated patterns were observed in different populations worldwide suggesting law-like behavior (Mitnitski et al., 2005; Rockwood and Mitnitski, 2006, 2011) which can be related to the fundamental processes of intrinsic biological aging in an organism. When the entire LLFS sample is characterized by deficits covering a wide spectrum of health dimensions these rates are robustly approximated by quadratic law. Exponential increase is more characteristic of more severe health dimensions (e.g., morbidity and disability).

Our results suggest that the aging rates approximated by the rates of changes in respective DIs are not altered by gender. At first glance this is surprising because it is widely believed that females have worse health than males although they live longer than males (Nathanson, 1975; Oksuzyan et al., 2008; Verbrugge, 1982). Consequently, females should accumulate more deficits than males over age. Various studies suggest, however, that gender differences may vary by health dimensions (see e.g., (Gorman and Read, 2006) and references therein). Furthermore, studies of age patterns of health deficits in the Framingham Heart Study population suggest presence of female and male specific health dimensions associated with cumulative effect of minor-effect traits (Kulminski et al., 2008a). As a result, females are found to accumulate more deficits than males when female-specific health dimensions are considered and vice versa. Given the wide spectrum of health dimensions such opposite trends for males and females can result in no gender differences (Kulminski et al., 2008a). 
Our results show that healthy individuals (i.e., those who contracted neither one of 21 major diseases listed in Table 1) can accumulate deficits in about the same (Fig. 2A) or even in more accelerated (Fig. 3A) fashion compared to unhealthy individuals. In each case, however, the shape of the age patterns was the same, i.e., quadratic for DI58 and exponential for DI27. Of importance is that faster aging rates for healthier individuals were also documented in other studies (Kulminski et al., 2006; Mitnitski et al., 2005). The differences in age dynamics between more and less healthy samples in those studies were, however, considerably more pronounced.

Disability status appears to be crucial for characterization of the aging rates. The age patterns for non-disabled individuals are likely the result of a convolution of different age-specific dynamics. This can be better understood when more severe health dimensions are considered (DI27). For instance, exponential pattern for disabled individuals in Fig. 3B (and Fig. 2B) is likely the result of: (i) exponential increase of disease-related deficits at younger ages (Fig. 3B, non-disabled), (ii) diminishing their role at older ages (Fig. 3B, non-disabled), (iii) non-essential role of disability-related deficits early in life (Fig. 3A, healthy), and (iv) exponential increase of disability-related deficits at older ages (Fig. 3A, healthy).

An interesting result is diminishing role of severe deficits in Fig. 3B for non-disabled individuals at advanced ages. Given that DI27 for non-disabled individuals characterizes co-morbidity, this pattern can decelerate because: (i) individuals at older ages are less fitted and, thus, they can be tolerant to smaller number of concurrent diseases than at younger ages or/and (ii) incidence of geriatric diseases can decline at advanced ages (see, e.g., Kamangar et al., 2006; Lerner and Kannel, 1986; Schoenberg et al., 1987).

The members of the LLFS study residing in Denmark, Boston, New York, and Pittsburg exhibit similar patterns of health deterioration. The oldest-old Danes aged 90-94 years can have, however, higher levels of disability and morbidity (characterized by DI27) compared to New York residents of the same age. Importantly, overall health status of centenarians residing in New York appears to be better compared to Boston residents.

At a first glance, lack of difference in the aging rates as characterized by the DI's age patterns for individuals selected for exceptional longevity and for their spouses, who should represent a general (control) population, is surprising. Indeed, it would be natural to expect differences in the aging rates for genetically unrelated individuals in the LLFS because DIs are good predictors of longevity (Kulminski et al., 2008b) whereas longevity shows modest heritability, i.e., about 25-35\% (Cournil et al., 2000; Gudmundsson et al., 2000; Herskind et al., 1996; Ljungquist et al., 1998; Skytthe et al., 2003). Furthermore, prior studies show that numerous health traits tend to be inherited in the LLFS (Matteini et al., 2010) and in other studies. For instance, offspring from the long-lived families in the Leiden Longevity Study had more favorable lipid profile, glucose tolerance, and hypertensive status (Rozing et al., 2010; Vaarhorst et al., 2010; Westendorp et al., 2009). Ashkenazi Jewish probands with exceptional longevity and their offspring also show better lipid profile compared to controls (Barzilai et al., 2003). Typically, centenarians have also little history of major aging-related diseases as, for instance, cardiovascular diseases and cancer (Barzilai et al., 2003; Willcox et al., 2008). Nevertheless, the same Leiden Longevity Study found no differences in some other health dimensions in long-living individuals, as, for instance, in hematopoietic capacity (Willems et al., 2008). Not all centenarians escaped major aging-related diseases (Engberg et al., 2009; Evert et al., 2003).

These findings suggest that offspring of long living individuals should not necessarily have better health in all possible health dimensions. This is actually in line with quite modest trend on better health (as characterized by the most comprehensive deficit index) of children of probands compared to controls, i.e., beta $=0.45$, in the age-aggregated sample. Although this trend was highly significant $\left(p=2.2 \times 10^{-5}\right)$, the effect size might be not sufficient to result in well pronounced differences in aging rates.

Furthermore, we should keep in mind at least three important considerations. First, inadequate sample size and lack of data at old ages (75+ years) prevents more detail comparison of the DIs age patterns at that age range. It might well be that aging rates for offspring and their spouses can diverge at more advanced ages. This would be an important observation which eventually implicates compression of morbidity. Second, in general, intrinsic (basal) aging rate for both long- and normal-living individuals can be the same even at advanced ages. Then, difference in lifespan of the normal- and long-living individuals can be due to weaker vulnerability of long-livers, i.e., due to their higher capacity coping with environmental stresses. Third, environmental changes occurring during lifespan of the same and different generations can greatly modulate the aging rates both directly and through interactions with genes resulting in so-called cohort effects and secular trends. Unfortunately, currently available data does not allow for testing the first two hypotheses. Third hypothesis looks interesting but it deserves separate analyses which are currently in progress.

Thus, we show that participants of the LLFS exhibit accelerated rates of aging following primarily quadratic pattern. These patterns are the same for males and females. Individuals contracted major diseases and those who were free of them exhibited the same aging rates as characterized by the DIs constructed using mild (no major diseases and disability) deficits. Unlike health, disability status can qualitatively change aging patterns. There are systemic differences in health among centenarians residing in New York and Boston.

\section{Role of the funding source}

This work was supported by National Institute on Aging (NIA) grants U01AG023746 and R01AG032319. The content is solely the responsibility of the authors and does not necessarily represent the official views of the NIA or the National Institutes of Health.

\section{References}

Barzilai, N., Atzmon, G., Schechter, C., Schaefer, E.J., Cupples, A.L., Lipton, R., Cheng, S., Shuldiner, A.R., 2003. Unique lipoprotein phenotype and genotype associated with exceptional longevity. JAMA 290, 2030-2040.

Cournil, A., Legay, J.M., Schachter, F., 2000. Evidence of sex-linked effects on the inheritance of human longevity: a population-based study in the Valserine valley (French Jura), 18-20th centuries. Proc. Biol. Sci. 267, 1021-1025.

Engberg, H., Oksuzyan, A., Jeune, B., Vaupel, J.W., Christensen, K., 2009. Centenarians - a useful model for healthy aging? A 29-year follow-up of hospitalizations among 40,000 Danes born in 1905. Aging Cell 8, 270-276.

Evert, J., Lawler, E., Bogan, H., Perls, T., 2003. Morbidity profiles of centenarians: survivors, delayers, and escapers. J. Gerontol. A Biol. Sci. Med. Sci. 58, 232-237.

Fried, L.P., Xue, Q.L., Cappola, A.R., Ferrucci, L., Chaves, P., Varadhan, R., Guralnik, J.M., Leng, S.X., Semba, R.D., Walston, J.D., Blaum, C.S., Bandeen-Roche, K., 2009. Nonlinear multisystem physiological dysregulation associated with frailty in older women: implications for etiology and treatment. J. Gerontol. A Biol. Sci. Med. Sci. 64, 1049-1057.

Goggins, W.B., Woo, J., Sham, A., Ho, S.C., 2005. Frailty index as a measure of biological age in a Chinese population. J. Gerontol. A Biol. Sci. Med. Sci. 60, 10461051.

Gorman, B.K., Read, J.G., 2006. Gender disparities in adult health: an examination of three measures of morbidity. J. Health Soc. Behav. 47, 95-110.

Gu, D., Dupre, M.E., Sautter, J., Zhu, H., Liu, Y., Yi, Z., 2009. Frailty and mortality among Chinese at advanced ages. J. Gerontol. B Psychol. Sci. Soc. Sci. 64, 279289.

Gudmundsson, H., Gudbjartsson, D.F., Frigge, M., Gulcher, J.R., Stefansson, K., 2000. Inheritance of human longevity in Iceland. Eur. J. Hum. Genet. 8, 743-749.

Herskind, A.M., McGue, M., Holm, N.V., Sorensen, T.I.A., Harvald, B., Vaupel, J.W. 1996. The heritability of human longevity: a population-based study of 2872 Danish twin pairs born 1870-1900. Hum. Genet. 97, 319-323.

Kamangar, F., Dores, G.M., Anderson, W.F., 2006. Patterns of cancer incidence, mortality, and prevalence across five continents: defining priorities to reduce 
cancer disparities in different geographic regions of the world. J. Clin. Oncol. 24, 2137-2150.

Katz, S., Akpom, C.A., 1976. 12. Index of ADL. Med. Care 14, 116-118.

Kulminski, A., Yashin, A., Ukraintseva, S., Akushevich, I., Arbeev, K., Land, K., Manton, K., 2006. Accumulation of health disorders as a systemic measure of aging: findings from the NLTCS data. Mech. Ageing Dev. 127, 840-848.

Kulminski, A., Yashin, A., Arbeev, K., Akushevich, I., Ukraintseva, S., Land, K., Manton, K., 2007a. Cumulative index of health disorders as an indicator of agingassociated processes in the elderly: results from analyses of the National Long Term Care Survey. Mech. Ageing Dev. 128, 250-258.

Kulminski, A.M., Ukraintseva, S.V., Akushevich, I.V., Arbeev, K.G., Yashin, A.I., 2007b Cumulative index of health deficiencies as a characteristic of long life. J Am Geriatr. Soc. 55, 935-940.

Kulminski, A.M., Culminskaya, I.V., Ukraintseva, S.V., Arbeev, K.G., Land, K.C., Yashin, A.I., 2008a. Sex-specific health deterioration and mortality: the morbiditymortality paradox over age and time. Exp. Gerontol. 43, 1052-1057.

Kulminski, A.M., Ukraintseva, S.V., Culminskaya, I.V., Arbeev, K.G., Land, K.C., Akushevich, L., Yashin, A.I., 2008b. Cumulative deficits and physiological indices as predictors of mortality and long life. J. Gerontol. A Biol. Sci. Med. Sci. 63, 1053-1059.

Lawton, M.P., Brody, E.M., 1969. Assessment of older people: self-maintaining and instrumental activities of daily living. Gerontologist 9, 179-186.

Lerner, D.J., Kannel, W.B., 1986. Patterns of coronary heart disease morbidity and mortality in the sexes: a 26-year follow-up of the Framingham population. Am. Heart J. 111, 383-390.

Ljungquist, B., Berg, S., Lanke, J., McClearn, G.E., Pedersen, N.L., 1998. The effect of genetic factors for longevity: a comparison of identical and fraternal twins in the Swedish Twin Registry. J. Gerontol. A Biol. Sci. Med. Sci. 53, M441-446.

Matteini, A.M., Fallin, M.D., Kammerer, C.M., Schupf, N., Yashin, A.I., Christensen, K., Arbeev, K.G., Barr, G., Mayeux, R., Newman, A.B., Walston, J.D., 2010. Heritability estimates of endophenotypes of long and health life: the long life family study. J. Gerontol. A Biol. Sci. Med. Sci..

Mitnitski, A.B., Mogilner, A.J., Rockwood, K., 2001. Accumulation of deficits as a proxy measure of aging. ScientificWorldJournal 1, 323-336.

Mitnitski, A., Song, X., Skoog, I., Broe, G.A., Cox, J.L., Grunfeld, E., Rockwood, K., 2005 Relative fitness and frailty of elderly men and women in developed countries and their relationship with mortality. J. Am. Geriatr. Soc. 53, 2184-2189.

Mitnitski, A., Bao, L., Skoog, I., Rockwood, K., 2007. A cross-national study of transitions in deficit counts in two birth cohorts: Implications for modeling ageing. Exp. Gerontol. 42, 241-246.

Nathanson, C.A., 1975. Illness and the feminine role: a theoretical review. Soc. Sci. Med. 9, 57-62.

Newman, A.B., Glynn, N.W., Taylor, C.A., Sebastiani, P., Perls, T.T., Mayeux, R., Christensen, K., Zmuda, J.M., Barral, S., Lee, J.H., Simonsick, E.M., Walston, J.D., Yashin, A.I., Hadley, E., 2011. Health and function of participants in the Long Life Family Study: a comparison with other cohorts. Aging (Albany NY) 3, 63-76.

Oksuzyan, A., Juel, K., Vaupel, J.W., Christensen, K., 2008. Men: good health and high mortality. sex differences in health and aging. Aging Clin. Exp. Res. 20, 91-102.

Pedersen, C.B., Gotzsche, H., Moller, J.O., Mortensen, P.B., 2006. The Danish civil registration system. A cohort of eight million persons. Dan. Med. Bull.53, 441-449.
Rockwood, K., Mitnitski, A., 2006. Limits to deficit accumulation in elderly people. Mech. Ageing Dev. 127, 494-496.

Rockwood, K., Mitnitski, A., 2011. Frailty defined by deficit accumulation and geriatric medicine defined by frailty. Clin. Geriatr. Med. 27, 17-26.

Rockwood, K., Song, X., MacKnight, C., Bergman, H., Hogan, D.B., McDowell, I., Mitnitski, A., 2005. A global clinical measure of fitness and frailty in elderly people. CMAJ 173, 489-495.

Rockwood, K., Mitnitski, A., Song, X., Steen, B., Skoog, I., 2006. Long-term risks of death and institutionalization of elderly people in relation to deficit accumulation at age 70. J. Am. Geriatr. Soc. 54, 975-979.

Rozing, M.P., Westendorp, R.G., de Craen, A.J., Frolich, M., de Goeij, M.C., Heijmans, B.T., Beekman, M., Wijsman, C.A., Mooijaart, S.P., Blauw, G.J., Slagboom, P.E., van Heemst, D., 2010. Favorable glucose tolerance and lower prevalence of metabolic syndrome in offspring without diabetes mellitus of nonagenarian siblings: the Leiden longevity study. J. Am. Geriatr. Soc. 58, 564-569.

Schoenberg, B.S., Kokmen, E., Okazaki, H., 1987. Alzheimer's disease and other dementing illnesses in a defined United States population: incidence rates and clinical features. Ann. Neurol. 22, 724-729.

Sebastiani, P., Hadley, E.C., Province, M., Christensen, K., Rossi, W., Perls, T.T., Ash, A.S., 2009. A family longevity selection score: ranking sibships by their longevity, size, and availability for study. Am. J. Epidemiol. 170, 1555-1562.

Skytthe, A., Pedersen, N.L., Kaprio, J., Stazi, M.A., Hjelmborg, J.V., Iachine, I., Vaupel, J.W., Christensen, K., 2003. Longevity studies in GenomEUtwin. Twin Res. 6, $448-454$.

Vaarhorst, A.A., Beekman, M., Suchiman, E.H., van Heemst, D., Houwing-Duistermaat, J.J., Westendorp, R.G., Slagboom, P.E., Heijmans, B.T., 2010. Lipid metabolism in long-lived families: the Leiden Longevity Study. Age (Dordr)

Vaupel, J.W., 2010. Biodemography of human ageing. Nature 464, 536-542.

Verbrugge, L.M., 1982. Sex differentials in health. Public Health Rep. 97, 417-437.

Westendorp, R.G, van Heemst, D., Rozing M.P., Frolich, M., Mooijaart, S.P., Blauw, G.J., Beekman, M., Heijmans, B.T., de Craen, A.J., Slagboom, P.E., 2009. Nonagenarian siblings and their offspring display lower risk of mortality and morbidity than sporadic nonagenarians: the Leiden Longevity Study. J. Am. Geriatr. Soc. 57, 1634-1637.

Willcox, D.C., Willcox, B.J., Wang, N.C., He, Q., Rosenbaum, M., Suzuki, M., 2008. Life at the extreme limit: phenotypic characteristics of supercentenarians in Okinawa. J. Gerontol. A Biol. Sci. Med. Sci. 63, 1201-1208.

Willems, J.M., Trompet, S., Eline Slagboom, P., de Craen, A.J., Westendorp, R.G., 2008. Hematopoietic capacity and exceptional survival: the Leiden Longevity Study. J. Am. Geriatr. Soc. 56, 2009-2013.

Woo, J., Goggins, W., Sham, A., Ho, S.C., 2006. Public health significance of the frailty index. Disabil. Rehabil. 28, 515-521.

Yashin, A.I., Arbeev, K.G., Kulminski, A., Akushevich, I., Akushevich, L., Ukraintseva, S.V., 2007a. Cumulative index of elderly disorders and its dynamic contribution to mortality and longevity. Rejuvenation Res. 10, 75-86.

Yashin, A.I., Arbeev, K.G., Kulminski, A., Akushevich, I., Akushevich, L., Ukraintseva, S.V., 2007b. Health decline, aging and mortality: how are they related? Biogerontology 8, 291-302.

Yashin, A.I., Arbeev, K.G., Kulminski, A., Borecki, I., Christensen, K., Barmada, M., Hadley, E., Rossi, W., Lee, J.H., Cheng, R., Elo, I.T., 2010. "Predicting” parental longevity from offspring endophenotypes: data from the Long Life Family Study (LLFS). Mech. Ageing Dev. 131, 215-222. 